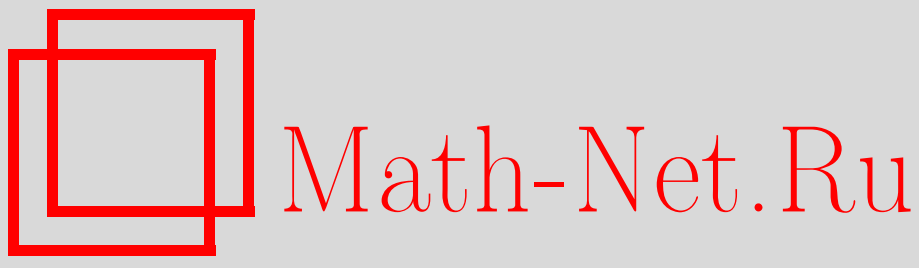

Г. Г. Ильюта, Геометрические реализации высших порядков Брюа и $M$ морсификации, Изв. РАН. Сер. матем., 1996, том 60, выпуск 6, 91-100

DOI: https://doi.org/10.4213/im96

Использование Общероссийского математического портала Math-Net.Ru подразумевает, что вы прочитали и согласны с пользовательским соглашением http: //www. mathnet.ru/rus/agreement

Параметры загрузки:

IP: 54.224 .135 .184

26 апреля 2023 г., 03:57:47 
УДК 513.5

\author{
Г. Г. Ильюта
}

\title{
Геометрические реализации высших поря,дков Брюа и $M$-морсификации
}

\begin{abstract}
Высшие порядки Брюа были введены Ю. И. Маниным и В. В. Шехтманом при изучении многомерных обобщений уравнения Янга-Бакстера. В статье представлена задача теории вещественных особенностей, которая обобщает исчисление змей В. И. Арнольда (кодирование связных компонент пространства очень хороших $M$-морсификаций особенности $\left.A_{n}\right)$ и в которой роль updown-перестановок играют их высшие аналоги - специального вида элементы высших порядков Брюа.

Библиографиия: 21 наименование.
\end{abstract}

Обычное в теории особенностей условие невырожденности морсификации критические значения различны - можно заменить другим, т.е. рассмотреть в пространстве параметров версальной деформации другую гиперповерхность в качестве дискриминанта. Основное содержание статьи составляет рассмотрение обобщенного страта Максвелла особенности $A_{n}$. Морсификащиями особенности $A_{n}$ являются полиномы от одной переменной степени $n+1$, и условие, что полином является общим (критические значения полинома различны), можно сформулировать следующим образом: никакие два экстремума графика морсификации не лежат на графике полинома степени 0, т.е. на графике константы. Наше обобщение ( $k$-обшие полиномы от одной переменной) состоит в рассмотрении следующего условия невырожденности морсификации: любое подмножество из $k+1$ элемента множества экстремумов графика морсификации не лежит на графике полинома степени $k-1$ (аналогично можно определить и более общий дискриминант, рассмотрев вместо графиков полиномов степени $k-1$ какое-либо другое $k$-мерное семейство кривых). В вещественном случае задача изучения $k$-страта Максвелла особенности $A_{n}$ обобщает исчисление змей В. И. Арнольда (кодирование связных компонент пространства очень хороших $M$-морсификаций особенности $\left.A_{n}\right)[2],[9]$.

Морсификация особенности называется максимальной (или $M$-морсификаuией), если число вещественных критических точек, родившихся из исходной вырожденной критической точки, максимально (равно кратности особенности). $M$-морсификация называется очень хорошей, если все ее критические значения различны [9]. Связные компоненты пространства очень хороших $M$-морсификаций особенности $A_{n}$ перечисляются updown-перестановками. В статье показано, что для $k$-вырождения роль updown-перестановок играют специального вида элементы высших порядков Брюа - обобшений симметрической группы, введенных Ю. И. Маниным и В. В. Шехтманом при изучении многомерного уравнения Янга-Бакстера [6]. 
Основные результаты теории $M$-морсификаций особенности $A_{n}$ состоят в рассмотрении двух взаимно однозначных отображений: набор связных компонент пространства очень хороших $M$-морсификаций отображается на множество ирdown-перестановок и пространство $M$-морсификаций гомеоморфно отображается на конус Спрингера $A_{n-1}[9]$. В статье строятся высшие аналоги этих отображений, что, в частности, позволяет определить высшие аналоги последовательности чисел Бернулли-Эйлера [2]. Также определяется высший аналог отображения Ляшко-Лойенги [1]. Статья является расширенным вариантом работы [5].

Наряду с изучением $k$-вырождения $M$-морсификаций (вешественных объектов) возникает аналогичная задача над полем комплексных чисел. Ниже мы покажем, что пространство $k$-общих наборов точек над $\mathbb{C}$ (которыми для $k$-обших полиномов будут наборы точек экстремума на графике полинома) является тотальным пространством расслоения, слоем которого является дополнение к дискриминантной конфигурации гиперплоскостей Манина-Шехтмана, а базой - дополнение к конфигурации группы кос Артина. Выписывание точной гомотопической последовательности этого расслоения дает следующий факт: пространство $k$-обших наборов точек над $\mathbb{C}$ является $K(\pi, 1)$-пространством, если и только если $K(\pi, 1)$-пространством является дополнение к дискриминантной конфигурации гиперплоскостей Манина-Шехтмана.

Опишем общую схемы (назовем ее схемой $A$ ) реализации высших порядков Брюа, частные случаи которой представлены в статье. Пусть даны множество $B$ и отображение из множества $k$-элементных подмножеств из $B$ в линейно упорядоченное множество $C$ (в рассмотренных ниже реализациях $B=C=\mathbb{R}$ ). Тогда при дополнительных условиях на вырождения упорядоченное $n$-элементное $(k \leqslant n)$ подмножество из $B$ задает линейньй порядок на $C(n, k)$-множестве $k$-элементных подмножеств из $\{1,2, \ldots, n\}$. Действительно, $k$-элементные подмножества упорядоченного $n$-элементного множества взаимно однозначно соответствуют элементам $C(n, k)$. Высшие порядки Брюа $B(n, k)$ являются подмножествами в множестве линейных порядков на $C(n, k)$, профакторизованными по некоторому отношению эквивалентности.

Приведем пример. $B(n, 1)$ совпадает с множеством перестановок чисел $1, \ldots, n$, иначе говоря, с множеством всех линейных порядков на $C(n, 1)=\{1, \ldots, n\}$. Рассмотрим множество из $n$ точек в $\mathbb{R}^{2}(x, y)$, у которых все координаты $x$ и все координаты $y$ различны (условие невырожденности). Точки упорядочены по величине координаты $x$ и по величине координаты $y$, что дает перестановку из $B(n, 1)$.

Условие $k$-обшности полинома является условием на набор точек экстремума его графика. Поэтому можно рассмотреть просто $k$-общие наборы точек, не обязательно являющиеся экстремумами графика некоторого полинома. Ниже описана реализация согласно схеме $A$ элементов высших порядков Брюа с помошњю конфигураций графиков полиномов степени $k-1$, строящихся по $k$-общим наборам точек в $\mathbb{R}^{2}$. В [6], [7] Ю. И. Манин и В. В. Шехтман описали реализацию элементов $B(n, k)$ с помощью конфигураций $n$ гиперплоскостей в $\mathbb{R}^{k}$. Для $k=2$ эти конструкции двойственны - конфигурация $n$ прямых в $\mathbb{R}^{2}$ двойственна конфигурации $n$ точек. Для $k>2$ конструкции расходятся. В п. 2 описана реализация схемы $A$ на таком уровне общности, который позволяет включить в одно семейство реализации элементов высших порядков Брюа с помощью графиков полиномов в $\mathbb{R}^{2}$ и 
конструкцию, двойственную конструкции Ю. И. Манина и В.В.Шехтмана.

Конфигурации прямых реализуют не все элементы $B(n, 2)$, а конфигурации псевдопрямых - все [3]. Где проходит граница между множествами конфигураций кривых, которых или достаточно или недостаточно для реализации всех элементов $B(n, 2)$ ? Аналогичный вопрос можно задать по отношению к $B(n, k)$.

В последнем пункте работы представлены некоторые вариации основной темы, к которым автор надеется вернуться в дальнейшем.

1. Определение высших порядков Брюа [6], [7], [14]. Через $C(n, k)$ обозначим множество $k$-элементных подмножеств в $\{1, \ldots, n\}$. Для $e \in C(n, k+1)$ назовем пакетом в $C(n, k)$ множество $\{d: d \in C(n, k), d \subset e\}$. Например, единственный пакет в $C(n, n-1)$ совпадает с $C(n, n-1)$. На $C(n, k)$ имеется естественный линейный порядок - лексикографический. Произвольный линейный порядок на $C(n, k)$ назовем допустимымм, если на каждом пакете он индуцирует либо лексикографический порядок, либо обратный лексикографическому. В частности, любой линейный порядок на $C(n, 1)$ является допустимым и множество линейных порядков на $C(n, 1)$ совпадает с множеством перестановок на $\{1, \ldots, n\}$. Через $A(n, k)$ обозначим множество всех допустимых порядков на $C(n, k)$. Два порядка из $A(n, k)$ называются әлементарно әквивалентнымии, если они отличаются только в паре соседних элементов, которые не принадлежат одному пакету. Фактор $A(n, k)$ по отношению эквивалентности, порожденному элементарной эквивалентностью, называется высшим порядком Брюа $B(n, k)$ (частичный порядок на $B(n, k)$ нам не понадобится, его определение можно найти в [14]).

2. Определение $k$-общих наборов точек. Пусть $F=\mathbb{R}$ или $F=\mathbb{C}$. Рассмотрим $F^{m+2}$ с декартовыми координатами $\left(x, y, z_{1}, \ldots, z_{m}\right)$. Набор из $n$ различных точек в $F^{m+2}$ назовем $k$-общим, $k+m \leqslant n$, если выполнены условия:

1) для любых $k+m$ точек набора $\left(x_{1}, y_{1}, z_{11}, \ldots, z_{m 1}\right), \ldots,\left(x_{m+k}, y_{m+k}\right.$, $\left.z_{1 m+k}, \ldots, z_{m m+k}\right)$

$$
\left|\begin{array}{cccccccc}
x_{1}^{k-1} & x_{1}^{k-2} & \ldots & x_{1} & 1 & z_{11} & \ldots & z_{m 1} \\
\ldots \ldots \ldots & \ldots \ldots \ldots \ldots \ldots & \ldots & \ldots & \ldots & \ldots & \ldots & \ldots \ldots \ldots \\
x_{m+k}^{k-1} & x_{m+k}^{k-2} & \ldots & x_{m+k} & 1 & z_{1} m+k & \ldots & z_{m m+k}
\end{array}\right| \neq 0
$$

2) координаты $x$ точек конфигурации различны;

3) любое $(m+k+1)$-элементное подмножество набора не лежит на графике полинома вида

$y=a_{k-1} x^{k-1}+\cdots+a_{1} x+a_{0}+b_{1} z_{1}+\cdots+b_{m} z_{m}, \quad a_{k-1}, \ldots, a_{0}, b_{1}, \ldots, b_{m} \in F$.

Из определения следует, что любому $k$-общему набору из $n$ точек можно сопоставить $\left(\begin{array}{c}n \\ m+k\end{array}\right)$ полиномов вида (1): каждому подмножеству из $m+k$ точек набора сопоставляется единственньй полином вида (1), на графике которого лежат эти точки. Назовем $k$-общий набор из $n$ точек вполне $k$-общuм, если коэффициенты при $(k-1)$-й степени $x$ сопоставленных набору полиномов различны.

Если точки набора упорядочены, то любому подмножеству из $k+m$ точек набора взаимно однозначно соответствует элемент множества $C(n, k+m)$. Поэтому полиномы $k$-общего набора упорядоченных точек будем обозначать через $P_{d}$, 
$d \in C(n, k+m)$, а их коэффишиенты при $(k-1)$-й степени $x-$ через $p_{d}$. Для $F=\mathbb{R}$ всегда будем считать, что точки $k$-общего набора упорядочены по возрастанию координаты $x$, и обозначим через $U(n, k)(V(n, k))$ пространство всех $k$-общих (вполне $k$-общих) упорядоченных наборов из $n$ точек.

Далее до конща статьи рассматривается частный случай конструкции этого пункта: $m=0$. В этом случае условие 1 ) в определении $k$-общего набора вытекает из условия 2), так как определитель Вандермонда не равен нулю. Поэтому набор $\left(x_{1}, y_{1}\right), \ldots,\left(x_{n}, y_{n}\right)$ является $k$-общим, если и только если $x_{i}$ различны и (условие 3$))$ для любого $(k+1)$-элементного подмножества $\left(x_{i_{1}}, y_{i_{1}}\right), \ldots,\left(x_{i_{k+1}}, y_{i_{k+1}}\right)$

$$
\left|\begin{array}{ccccc}
y_{i_{1}} & x_{i_{1}}^{k-1} & \ldots & x_{i_{1}} & 1 \\
\ldots \ldots & \ldots \ldots \ldots \ldots & \ldots \ldots \\
y_{i_{k+1}} & x_{i_{k+1}}^{k-1} & \ldots & x_{i_{k+1}} & 1
\end{array}\right| \neq 0 .
$$

3. Фундаментальная группа пространства $k$-общих наборов над $\mathbb{C}$. Напомним определение дискриминантной конфигурации гиперплоскостей Манина-Шехтмана [14]. Пусть $F$ - поле и $H_{1}^{0}, \ldots, H_{n}^{0} \subset F^{k}$ - семейство аффинных гиперплоскостей в общем положении. Через $D(n, k)$ обозначим пространство конфигураций гиперплоскостей $H_{1}, \ldots, H_{n} \subset F^{k}$, обладающих свойствами: $H_{i}$ параллельно $H_{i}^{0}$ для всех $i ; H_{1}, \ldots, H_{n}$ находятся в общем положении. $D(n, k)$ является подмножеством в пространстве $F^{n}$ всех параллельных сдвигов гиперплоскостей $H_{i}^{0}$. Более того, $F^{n} \backslash D(n, k)$ есть конфигурация гиперплоскостей следующего вида. Для $d \in C(n, a)$ положим

$$
D_{d}=\left\{\left(H_{1}, \ldots, H_{n}\right) \in F^{n}: \bigcap_{i \in d} H_{i} \neq \varnothing\right\} .
$$

Ясно, что $D_{d}=F^{n}$, если $k \geqslant a$ и $\operatorname{codim} D_{d}=a-k, a \geqslant k+1$.

В частности, $D_{d}$ для $d \in C(n, k+1)$ являются попарно различными гиперплоскостями в $F^{n}$ и

$$
D(n, k)=F^{n} \backslash \bigcup_{d \in C(n, k+1)} D_{d} .
$$

Конфигурация гиперплоскостей $D_{d}, d \in C(n, k+1)$, называется дискриминантной конфигурачией Манина-Шехтмана.

Приведем также определение циклической конфигурации гиперплоскостей [21]. Пусть $x_{1}, \ldots, x_{n}$ - множество различных элементов поля $F$. Циклической конфиәурацией называется множество гиперплоскостей

$$
Z_{i}=\left\{\left(z_{1}, \ldots, z_{k}\right) \in F^{k}: z_{1}+x_{i} z_{2}+\cdots+x_{i}^{k-1} z_{k}+x_{i}^{k}=0\right\}
$$

Нетрудно видеть, что дополнение к дискриминантной конфигурации Манина-Шехтмана, строящейся по циклической конфигурации, задается условиями (2). Поэтому пространство $k$-общих наборов $U(n, k)$ расслоено над дополнением к конфигурации группы кос $E_{n}=F^{n} \backslash\left\{x_{i}=x_{j}, i<j\right\}$ и слоем является дополнение к дискриминантной конфигурации Манина-Шехтмана $D(n, k)$. Пусть $F=\mathbb{C}$. Тогда дополнение к конфигурации группы кос является $K(\pi, 1)$-пространством [1], и поэтому из точной гомотопической последовательности расслоения вытекает 
ПРЕДЛОЖЕНИЕ. Имеем КоротКую Точную последовательность

$$
0 \rightarrow \pi_{1}(D(n, k)) \rightarrow \pi_{1}(U(n, k)) \rightarrow \pi_{1}\left(E_{n}\right) \rightarrow 0
$$

и изоморфизмь

$$
\pi_{i}(D(n, k)) \approx \pi_{i}(U(n, k)), \quad i>1 .
$$

Таким образом, пространство $k$-общих наборов точек над $\mathbb{C}$ является $K(\pi, 1)$-пространством, если и только если $K(\pi, 1)$-пространством является дополнение к дискриминантной конфигурации Манина-Шехтмана.

4. Высшие порядки Брюа и $k$-общие наборы в $\mathbb{R}^{2}$. Далее рассматривается конструкция п. 2 для $m=0, F=\mathbb{R}$. Построим постоянные на компонентах связности отображения

$$
a: V(n, k) \rightarrow A(n, k), \quad b: U(n, k) \rightarrow B(n, k) .
$$

Лемма 1. Рассмотрим к-общий набор из $n$ точек и два полинома этого набора, $P_{d}, P_{q}, d, q \in C(n, k)$. Если $p_{d}=p_{q}$, mо $d, q$ не принадлежат одному пакету.

Действительно, разность $P_{d}-P_{q}$ имеет не более чем $k-2$ корня, т.е. $d, q$ имеют не более чем $k-2$ общих элемента, и поэтому не могут принадлежать одному пакету.

Согласно лемме 1 для любого набора из $U(n, k)$ можно упорядочить по величине числа $p_{d}$, отвечающие произвольному пакету в $C(n, k)$, и тем самым получить порядок на произвольном пакете.

Ключевым является следуюшее

ПРЕДЛОЖЕНИЕ 1. Полученный порядок на любом пакете является либо лексикографическим, либо обратным лексикографическому.

Вначале отметим, что тем самым определено $a$, а именно для любого набора из $V(n, k)$ упорядочиваем по величине числа $p_{d}, d \in C(n, k)$, что дает порядок на $C(n, k)$, который в силу предложения 1 принадлежит $A(n, k)$. Определено также и $b$ : если какие-то из чисел $p_{d}$ совпадают, то все возможные упорядочения этих чисел приводят согласно лемме 1 к эквивалентным порядкам из $A(n, k)$, т.е. к одному и тому же элементу из $B(n, k)$. Очевидно, что $a$ постоянно на компонентах связности пространства $V(n, k)$, и из леммы 1 следует, что $b$ постоянно на компонентах связности пространства $U(n, k)$.

ДОКАЗАТЕЛЬСТВО ПРЕДЛОЖЕНИЯ 1. УПорядочим отвечающие пакету точки $A_{1}, \ldots, A_{k+1}$ по возрастанию координаты $x$. Через $P_{i}$ обозначим полином набора, график которого не содержит $A_{i}$ и содержит остальные точки пакета, $p_{i}$ - старший коэффициент полинома $P_{i}$. Согласно лемме 1 числа $p_{i}$ различны. Каждая пара графиков полиномов $P_{i}$ имеет $k-1$ общую точку среди $A_{1}, \ldots, A_{k+1}$, и степени полиномов $P_{i}$ не превосходят $k-1$. Поэтому все точки пересечения графиков полиномов $P_{i}$ принадлежат $\left\{A_{1}, \ldots, A_{k+1}\right\}$.

График полинома делит $\mathbb{R}^{2}$ на две части, и поэтому можно говорить о точках, расположенных вьше и ниже графика. При больших $x$ график $P_{i}$ расположен вьше 
графика $P_{j}$, если и только если $p_{i}>p_{j}$. Пусть $A_{k+1}$ расположена выше графика $P_{k+1}$. Покажем, что $p_{1}>\cdots>p_{k+1}$ (если $A_{k+1}$ расположена ниже графика $P_{k+1}$, то совершенно аналогично доказывается, что $\left.p_{1}<\cdots<p_{k+1}\right)$. Так как все точки пересечения графиков принадлежат $\left\{A_{1}, \ldots, A_{k+1}\right\}$, то $p_{k}>p_{k+1}$. Между точками $A_{k-1}$ и $A_{k+1}$ график $P_{k}$ выше графика $P_{k+1}$, и поэтому между точками $A_{k-2}$ и $A_{k}$ график $P_{k-1}$ ниже графиков $P_{k}$ и $P_{k+1}$, и тем самым при больших $x$ график $P_{k-1}$ выше графиков $P_{k}$ и $P_{k+1}$, т.е. $p_{k-1}>p_{k}>p_{k+1}$. Аналогично для всех $i$ : если между точками $A_{k-i}$ и $A_{k-i+2}$ график $P_{k-i+1}$ выше (ниже) графиков $P_{k-i+2}, \ldots, P_{k+1}$, то между точками $A_{k-i-1}$ и $A_{k-i+1}$ график $P_{k-i}$ ниже (выше) графиков $P_{k-i+1}, \ldots, P_{k+1}$, и поэтому при больших $x$ график $P_{k-i}$ выше графиков $P_{k-i+1}, \ldots, P_{k+1}$, т.e. $p_{k-i}>\cdots>p_{k+1}$.

5. Updown-элементы высших порядков Брюа и $k$-общие полиномы. Перенесем конструкции п. 4 на $k$-общие наборы точек, которые появляются как множества точек экстремума на графиках полиномов. Назовем полином над $F$ степени $n+1$ со старшим коэффициентом 1 (вполне) $k$-общим, если он имеет $n$ различных критических точек в $F$ и множество точек экстремума его графика образует (вполне) $k$-общий набор. Критические значения $k$-обшего полинома над $\mathbb{R}$ образуют updown-последовательность: $y_{n}<y_{n-1}>y_{n-2}<\cdots$. Это мотивирует следующие определения.

Для $F=\mathbb{R}$ назовем (вполне) $k$-общий набор из $n$ точек updown-набором, если координаты $y$ точек набора образуют updown-последовательность. Обозначим через $\mathrm{Up}(n, k)$ и $\operatorname{Vp}(n, k)$ пространства всех updown-наборов в $U(n, k)$ и $V(n, k)$. Образ отображения, сопоставляющего (вполне) $k$-общему полиному степени $n+1$ набор точек экстремума его графика, принадлежит $(\mathrm{Vp}(n, k)) \operatorname{Up}(n, k)$.

Элемент $d \in A(n, k)$ назовем updown-элементом, если выполнено следующее условие. Рассмотрим последовательность пакетов в $C(n, k)$, отвечающих следуюшим элементам $C(n, k+1):(1, \ldots, k+1),(2, \ldots, k+2), \ldots,(n-k, \ldots, n)$. Элемент $d$ индуцирует полные порядки на этих пакетах, и мы потребуем, чтобы лексикографический и обратный лексикографическому порядки в последовательности пакетов чередовались, причем на последнем пакете порядок обратный лексикографическому. Для $k=1$ получается обычное определение updown-перестановки [2]. Из определения очевидно, что весь класс эквивалентности updown-элемента состоит из updown-элементов. Соответствующие элементы $B(n, k)$ также назовем updown-элементами. Обозначим через $\operatorname{Ap}(n, k)(\mathrm{Bp}(n, k))$ множество updown-элементов в $A(n, k)(B(n, k))$. Через ap $(b p)$ обозначим ограничение отображения $a(b)$ на $\mathrm{Vp}(n, k) \quad(\mathrm{Up}(n, k))$. Легко видеть, что $a p(b p)$ постоянно на компонентах связности пространства $\operatorname{Vp}(n, k)(\mathrm{Up}(n, k))$.

ПРЕДЛОЖЕНИЕ 2. Образ отображения ар $(b p)$ лежит в $\operatorname{Ap}(n, k)(\operatorname{Bp}(n, k))$.

ДокАЗАТЕльСТво. Если $i$-я точка набора является точкой локального максимума (минимума), т.е. $y_{i-1}<y_{i}>y_{i+1}\left(y_{i-1}>y_{i}<y_{i+1}\right)$, то она лежит вьше (ниже) графика полинома, содержащего точки с номерами $i-k, \ldots, i-1$. Это вытекает из того, что $y_{i-1}<y_{i-2}>\cdots\left(y_{i-1}>y_{i-2}<\cdots\right)$, и следующего замечания: полином, график которого содержит точки с номерами $i-k, \ldots, i-1$, имеет степень не более $k-1$, и поэтому его производная меняет знак не более $k-2$ раз. Поэтому на пакете, отвечающем точкам $(i-k, \ldots, i)$, порядок лексикографический 
(обратный лексикографическому) согласно предложению 1.

ГИПоТЕЗА 1. Отображения $a, b, a p, b p$ индуцируют биекиии своих образов $u$ множсеств связных компонент пространств $V(n, k), U(n, k), \mathrm{Vp}(n, k)$, $\mathrm{Up}(n, k)$.

ГИПоТЕЗА 2. Если әлемент $B(n, k)(\mathrm{Bp}(n, k))$ принадлежит образу отображения $b(b p)$, то весь прообраз этого элемента при факторизации в $A(n, k)$ $(\operatorname{Ap}(n, k))$ принадлежст образу отображения а $($ ар).

6. Обобщенное отображение Ляшко-Лойенги. Построим высший аналог отображения Ляшко-Лойенги и отображения $M$-морсификаций $A_{n}$ в конус Спрингера $A_{n-1}[1],[9]$. Занумеруем в лексикографическом порядке элементы $C(n, k)$ и согласно номерам сопоставим их координатным функциям $z_{i}$ в $F^{m}$, $m=\left(\begin{array}{l}n \\ k\end{array}\right)$. Обозначим через $G(n, k)$ конфигурацию группы кос $\left\{z_{i}=z_{j}, i<j\right\}$ в $F^{m}$. Через $H(n, k)$ обозначим конфигурацию гиперплоскостей в $F^{m}$, которая получается, если из конфигурации $G(n, k)$ выбросить те гиперплоскости $z_{i}=z_{j}$, для которых отвечающие координатам $z_{i}, z_{j}$ элементы $C(n, k)$ не принадлежат одному пакету. Согласно лемме для любого (вполне) $k$-общего набора из $n$ точек вектор чисел $p_{d}, d \in C(n, k)$, принадлежит $\left(F^{m} \backslash G(n, k)\right) F^{m} \backslash H(n, k)$. Для $F=\mathbb{R}$ согласно предложению 1 образ построенного отображения принадлежит $(T(n, k) \backslash G(n, k)) T(n, k) \backslash H(n, k)$, где $T(n, k) \subset \mathbb{R}^{m}$ определяется как множество всех точек, координаты которых удовлетворяют условию: подмножество координат $z_{i_{1}}, \ldots, z_{i_{k+1}}, i_{1}<\cdots<i_{k+1}$, отвечающих любому пакету в $C(n, k)$, является решением одной из систем неравенств: $z_{i_{1}}>\cdots>z_{i_{k+1}}$ или $z_{i_{1}}<\cdots<z_{i_{k+1}}$.

Определим $k$-конус Спрингера $S(n, k) \subset T(n, k)$ дополнительным условием: любое подмножество координат $z_{i_{1}}, \ldots, z_{i_{k+1}}$, отвечающее пакету элемента $(q-k, \ldots, q+1) \in C(n, k)$, является решением системы $z_{i_{1}} \geqslant \cdots \geqslant z_{i_{q+1}}$, если $n-q$ четно, и решением системы $z_{i_{1}} \leqslant \cdots \leqslant z_{i_{q+1}}$, если $n-q$ нечетно. Согласно предложению 2 сопоставление $k$-общему набору из $n$ точек вектора из чисел $p_{d}$, $d \in C(n, k)$, задает отображение в $k$-конус Спрингера

$$
\mathrm{Vp}(n, k) \rightarrow S(n, k) \backslash G(n, k), \quad \mathrm{Up}(n, k) \rightarrow S(n, k) \backslash H(n, k) .
$$

$F^{m}$ можно факторизовать по действию симметрической группы (перестановка координат). Сквозное отображение $\{$ полиномы $\} \rightarrow\{$ наборы экстремумов графиков полиномов $\} \rightarrow F^{m}$ и факторизация по действию симметрической группы обобшают отображение Ляшко-Лойенги.

7. Торическая точка зрения. В п. $4 k$-общему набору из $n$ точек в $\mathbb{R}^{2}$ сопоставляется $\left(\begin{array}{l}n \\ k\end{array}\right)$ полиномов и линейный порядок на $C(n, k)$ задается упорядочением по величине коэффициентов при $(k-1)$-й степени $x$ этих полиномов. Оказывается, что столь же хорошими свойствами обладают упорядочения коэффициентов этих полиномов при любом мономе $x^{m}, k-1>m \geqslant 0$, но, как обычно в торической геометрии, нужно потребовать, чтобы объекты “хорошо вели себя" по отношению к началу координат.

Из определения понятно, что, заменив порядок в допустимом линейном упорядочении элементов $C(n, k)$ на обратный, мы снова получим допустимый порядок. 
Также очевидно, что, заменив порядок на обратный в двух эквивалентных линейных порядках на $C(n, k)$, снова получим эквивалентные линейные порядки. Поэтому для любого элемента из $B(n, k)$ определен обратный ему элемент, также принадлежащий $B(n, k)$.

Рассмотрим $k$-общий набор из $n$ точек в $\mathbb{R}^{2}$, причем пусть координаты $x$ всех точек набора положительны. Покажем, что для $k-1>m \geqslant 0$ упорядочение по величине коэффициентов при $x^{m}$ полиномов набора задает тот же элемент $B(n, k)$, что и упорядочение коэффициентов при $x^{k-1}$, если $k-m$ нечетно, и обратный элемент, если $k-m$ четно. Это вытекает из леммы 2 и предложения 3 с учетом того, что любой элемент $B(n, k)$ определяется порядками на всех пакетах [14].

ЛЕмма 2. Пусть координаты $x$ всех точек $k$-общего набора положительны. Рассмотрим два полинома этого набора, $P_{d}, P_{q}, d, q \in C(n, k)$. Если коэффициенты при $x^{m}(k-1 \geqslant m \geqslant 0)$ этих полиномов совпадают, то $d, q$ не принадлежат одному пакету.

ДокАЗАТельСТво. Достаточно показать, что $P_{d}-P_{q}$ имеет не более $k-2$ положительных корней (сравните с леммой 1$)$. Так как коэффициент при $x^{m} \mathrm{y}$ полинома $P_{d}-P_{q}$ равен нулю, то это вытекает из правила Декарта (теоремы о малочленах) [8].

Приведем более прямое доказательство, которое понадобится в предложении 3. Если $d, q$ принадлежат одному пакету, то они имеют $k-1$ общий элемент, и поэтому у полинома степени не выше $k-1 P_{d}-P_{q}$ все корни вешественны и положительны. Тогда и все корни производных порядка $0,1, \ldots, k-1$ этого полинома вещественны и положительны (они лежат на отрезке, концами которого являются минимальный и максимальный корни полинома $\left.P_{d}-P_{q}\right)$. Но нуль является корнем $m$-й производной полинома, у которого коэффициент при $x^{m}$ равен нулю.

ПРЕДЛОЖЕНИЕ 3 . Пусть координаты $x$ всех точек $k$-общего набора из $n$ точек в $\mathbb{R}^{2}$ положительны. Тогда порядок на коэффичиентах при $x^{m}$ полиномов набора, отвечающих одному пакету, совпадает с порядком на коэффициентах при $x^{k-1}$, если $k-m$ нечетно, и с обратным этому порядку, если $k-m$ четно.

ДокАЗАТЕЛЬСТво. Как показано в доказательстве леммы 2 для пары полиномов $P_{d}, P_{q}$, отвечаюших одному пакету, у полинома $P_{d}^{(m)}-P_{q}^{(m)}$ все корни вещественны и положительны. Поэтому на положительной полуоси полиномы $P_{d}^{(m)}$ и $P_{q}^{(m)}$ “меняются местами" (их графики пересекаются) $k-m-1$ раз, т.е. для всех пар полиномов пакета их графики пересекаются одинаковое число раз. Коэффициент при $x^{k-1}$ полинома $P_{d}$ совпадает с коэффициентом при $x^{k-m-1}$ полинома $P_{d}^{(m)} /((k-1) \ldots(k-m))$. Остается заметить, что упорядочение по величине коэффициентов при $x^{m}$ полиномов $P_{d}$ совпадает с порядком на прямой $x=0$ точек пересечения этой прямой с графиками полиномов $P_{d}^{(m)}$, а упорядочение по величине коэффициентов при $x^{k-1}$ совпадает с порядком на прямой $x=c$, если $c$ достаточно велико.

8. Направления дальнейших исследований. В конструкции п. 2, включаюшей в одно семейство реализации элементов высших порядков Брюа с помошью графиков полиномов в $\mathbb{R}^{2}$ и конструкцию, двойственную конструкции 
Ю. И. Манина и В.В. Шехтмана [6], [7], используются конфигурации гиперповерхностей вида

$$
y=\sum_{i=0}^{k-1} a_{i} x^{i}+\sum_{i=1}^{m} b_{i} y^{i} .
$$

Такие гиперповерхности являются простейшими обобщениями гиперплоскостей определяюшее уравнение полиномиально по одной из переменных и линейно по остальным. Интересно было бы описать связи высших порядков Брюа и дискриминантных конфигураций Манина-Шехтмана с обобщениями на такие конфигурации гиперповерхностей основных конструкций теории конфигураций гиперплоскостей: алгебра Орлика-Соломона [17], комплекс Салветти [18], кольцо Варченко-Гельфанда [4], монодромия кос для $m=0$ [15], [16].

Пространство комплексных полиномов с нестандартным дискриминантом в нем рассматривалось в [10]. Полином называется лемнискатно общим [10] (или типичным [2]), если его критические значения не равны нулю и имеют разные модули. Назовем лемнискатно общий полином әллиптически общим, если его критические значения лежат на разных лучах с началом в нуле в плоскости значений полинома $\mathbb{C}$. Гиперболически общим назовем комплексньй полином, все критические значения которого лежат на разных гиперболах

$$
(\operatorname{Re} z)^{2}-(\operatorname{Im} z)^{2}=c \neq 0
$$

$(\operatorname{Re} z, \operatorname{Im} z-$ соответственно вещественная и мнимая части комплексного числа $z$, $c \in \mathbb{R})$ и имеют разные $a$ в естественной параметризации этих гипербол с помошью гиперболических синуса и косинуса $\operatorname{sh} a, \operatorname{ch} a$. Аналогично пп. 2, 3 можно определить эллиптически (гиперболически) $k$-общие полиномы с помощью (гиперболических) тригонометрических полиномов и реализовать для них схему $A$. По-видимому, на эллиптически и гиперболически $k$-общие полиномы могут быть обобщены результаты работы [10], в которой описана связь лемнискатно общих полиномов с $M$-морсификациями.

В стратификации пространства комплексных полиномов от одной переменной, задаваемой обычным стратом Максвелла (т.е. в стратификации по числу критических значений полинома), кроме общего страта довольно интенсивно изучался еще один - обобщенные полиномы Чебышева, т.е. полиномы, имеюшие не более двух критических значений [19]. Назовем $(m, n)$-полиномом Чебышева комплексньй полином, для которого сушествуют полином степени не вьше $m$ и полином степени не выше $n$, на графиках которых лежат все экстремумы графика исходного полинома.

Р.Стенли сопоставил стандартные таблицы Юнга линейным порядкам на $C(n, 2)$, связанным с высшим порядком Брюа $B(n, 2)[20]$. В [11] любому линейному порядку на $C(n, 2)$ сопоставлена таблица Юнга следуюшим образом: в клетке на пересечении $i$-го столбца и $j$-й строки $(i<j)$ таблицы записывается порядковый номер элемента $(i, j) \in C(n, 2)$ в линейном порядке. Описаны также таблищы (так называемые сбалансированные таблицы Юнга), отвечаюшие при таком отображении линейным порядкам на $C(n, 2)$, связанным с $B(n, 2)$, и алгоритм перехода от сбалансированных таблиц к стандартным. Определение из [11] очевидным образом обобщается на линейные порядки на $C(n, k)$ и многомерные таблищы 
Юнга. А именно, в клетке многомерной таблицы Юнга с координатами $i_{1}, \ldots, i_{k}$, $i_{1}<\cdots<i_{k},\left(i_{1}, \ldots, i_{k}\right) \subset\{1, \ldots, n\}$, записывается порядковый номер элемента $\left(i_{1}, \ldots, i_{k}\right) \in C(n, k)$ в линейном порядке.

\section{Список литературы}

1. Арнольд В.И., Варченко А.Н., Гусейн-Заде С. М. Особенности дифференцируемых отображений. М.: Наука, 1984.

2. Арнольд В. И. Исчисление змей и комбинаторика чисел Бернулли, Эйлера и Спрингера групп Кокстера // УМН. 1992. Т. 47. № 1. С. 3-45.

3. Воеводский B. A., Капранов M. M. Свободная $n$-категория, порожденная кубом, ориентированные матроиды и высшие порядки Брюа // Функц. анализ и его прилож. 1991. T. 25. № 3. C. $62-65$.

4. Гельфанд И. М., Рьббиков Г. Л. Алгебраические и топологические инварианты ориентированных матроидов // ДАН. 1989. Т. 307. № 4. С. 791-795.

5. Ильюта Г. Г. Высшие порядки Брюа и $M$-морсификации // ДАН. 1996. Т. 347. № 3. C. 306-308.

6. Манин Ю.И., Шехтман В.В. Расположения вещественных гиперплоскостей и уравнения Замолодчикова // Тр. семинара “Теоретико-групповые методы в физике”. 1986. T. 1. C. $316-324$.

7. Манин Ю.И., Шехтман В.В. О высших порядках Брюа, связанных с симметрической группой // Функц. анализ и его прилож. 1986. Т. 20. № 2. С. 74, 75.

8. Хованский А.Г. Об одном классе трансцендентных уравнений // ДАН СССР. 1980. T. 255. № 4. C. 804-807.

9. Arnold V. I. Springer number and morsification spaces // J. Algebraic Geometry. 1992. V. 1. № 2. P. 197-214.

10. Cataneze F., Frediani P. Configurations of real and complex polynomials // Asterisque. 1993. V. 218. P. 61-93.

11. Edelman P., Greene C. Balanced tableaux // Adv. Math. 1987. V. 63. P. 42-99.

12. Kirillov A. N., Berenstein A.D. Groups generated by involutions, Gelfand-Tsetlin patterns, and combinatorics of Young tableaux // Алгебра и анализ. 1995. Т. 7. № 1. C. $92-152$.

13. Looijenga E. The discriminant of a real simple singularity // Compositio Math. 1987. V. 37. Fase 1. P. 51-62.

14. Manin Y.I., Schechtman V.V. Arrangements of hyperplanes, higher braid groups and higher Bruhat orders // Adv. Studies in Pure Math. 1985. V. 44. P. 289-308.

15. Moishezon B. Algebraic surfaces and the arithmetic of braids. II // Contemporary Math. 1985. V. 44. P. 311-344.

16. Moishezon B., Teicher $M$. Braid group technique in complex geometry. I: Line arrangements in $\mathrm{CP}^{2}$ // Contemporary Math. 1986. V. 78. P. 425-555.

17. Orlik P., Terao H. Arrangements of hyperplanes. Berlin: Springer-Verlag, 1992.

18. Salvetti $M$. Topology of the complement of real hyperplanes in $\mathbb{C}^{n} / /$ Invent. Math. 1987. V. 88. P. 603-618.

19. Shabat G., Zvonkin A. Plane trees and algebraic numbers // Contemporary Math. 1994. V. 178. P. 233-275.

20. Stanley $R$. On the number of reduced decompositions of elements of Coxeter groups // European J. Combinatorics. 1984. V. 5. P. 359-372.

21. Ziegler G. M. Higher Bruhat orders and cyclic hyperplane arrangements // Topology. 1993. V. 32. № 2. P. 259-279. 